\title{
Issues Concerning High Current Lower Energy Electron Beams Required for Ion Cooling between EBIS LINAC and Booster
}

\author{
A. Hershcovitch
}

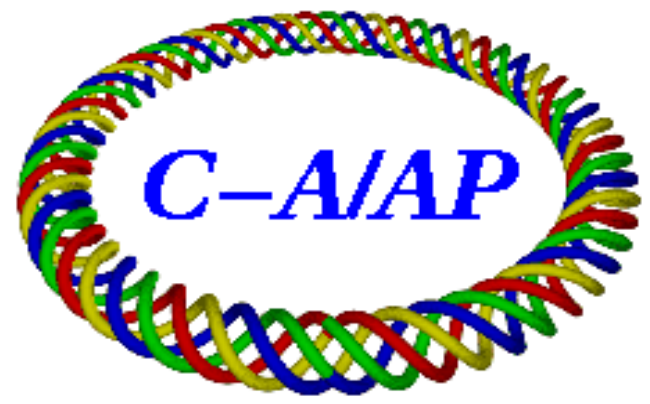

\section{Collider-Accelerator Department Brookhaven National Laboratory Upton, NY 11973}

Notice: This document has been authorized by employees of Brookhaven Science Associates, LLC under Contract No. DE-AC02-98CH10886 with the U.S. Department of Energy. The United States Government retains a nonexclusive, paid-up, irrevocable, world-wide license to publish or reproduce the published form of this document, or allow others to do so, for United States Government purposes. 


\title{
Issues Concerning High Current Low Energy Electron Beams Required for Ion Cooling between EBIS LINAC and Booster
}

\author{
Ady Hershcovitch
}

Some issues, regarding a low energy high current electron beam that will be needed for electron beam cooling to reduce momentum of gold ions exiting the EBIS LINAC before injection into the booster, are examined. Options for propagating such an electron beam, as well as the effect of neutralizing background plasma on electron and ion beam parameters are calculated. Computations and some experimental data indicate that none of these issues is a show stopper.

\section{Introduction}

It was suggested ${ }^{1}$ to use an electron beam for single-pass cooling of the ion beam end of the EBIS-RFQ-LINAC system. Expected ion beam parameters ${ }^{2}$, based on design, at the exit of the EBIS-RFQ-LINAC system are: energy $2 \mathrm{MeV} / \mathrm{u}$, momentum spread $\Delta p_{\|} / p=10^{-3}$, and $\Delta p_{\perp} / p=5 \times 10^{-3}$, beam diameter $1 \mathrm{~cm}$, and gold ion charge state $\mathrm{Au}^{+32}$, with ion density ${ }^{2}$ at the LINAC exit $\mathrm{n}_{\mathrm{i}}=8 \times 10^{7} \mathrm{~cm}^{-3}$. For electrons to match ion velocity, their energy $\mathrm{U}$ must be about $1 \mathrm{KeV}$ (actually slightly more than $1.098 \mathrm{KeV}$ ). At these energies, ion and electron velocities are about $2 \times 10^{7}$ meter/second, hence $\beta=$ 0.0667 and $\gamma=1.0022$.

In those basic cooling computation electron beam parameters based on what was obtained with an electron gun with plasma cathode, from which 9 A were extracted at $1 \mathrm{KeV}$ through a $6 \mathrm{~mm}$ aperture ${ }^{3-6}$. Based on these parameters the electron density $\mathrm{n}$ can be computed from $n=I / A e v$, where I is electron beam current, e elementary charge and A is extraction aperture area. Immediately after extraction, the electron density is about $n \approx 10^{11} \mathrm{~cm}^{-3}$ (electron beam current density of $31.8 \mathrm{~A} / \mathrm{cm}^{2}$ ). Balk electrons parallel energy spread before extraction was about $0.1 \mathrm{eV}$; measured perpendicular spread was under $0.5 \mathrm{eV}$ (limited by measurement resolution). To match ion beam diameter, i.e. fill $1 \mathrm{~cm}$ diameter at the obtained ${ }^{3-6}$ electron current density, a 25 A electron beam is needed.

Analyzed cooling scenario ${ }^{1}$ is based on magnetized cooling in a 2.4 Tesla solenoidal magnetic field. And obviously, cooling is to be performed in vacuum; most likely in tubular vacuum system. Issues concerning propagation of magnetically confined electron beams through pipes have been described in a number of text books ${ }^{7,8}$. As it was pointed out $^{9}$, injection of $1 \mathrm{KeV}, 25 \mathrm{Amp}$ electron beam through such a system cannot result in beam propagation that has both $1 \mathrm{KeV}$ electron energy and , $25 \mathrm{Amp}$ electron current.

Two possible solutions are analyzed in this note: space charge neutralization and injection at higher energies. The first is simple analytically, but requires calculations of multiple effects. The later is analytically complex, but seems at first pass easier to 
implement experimentally. Either solution indicates that none of the issues to be raised in subsequent sections is a "show-stopper."

\section{Space Charge Neutralization}

Simplest way to neutralize the electron beam is by gas or plasma whose density equals (or is greater than) electron beam density. Electron beam space charge neutralization requires introduction of gas and/or plasma, since the ion beam charge density is a factor of 39 lower than the electron beam charge density. With only the ion beam, electron beam neutralization is $2.56 \%$. But, neutralizing plasma and/or gas particles scatter beam ions or electrons. In this case of interest with target thickness of only $\mathrm{nl} \approx 10^{13} \mathrm{~cm}^{-2}$ effects on the beam envelope (i.e. significant spatial scattering) can be neglected (especially in a 2.4 Tesla magnetic field). However, velocity space diffusion and slowing down must be calculated.

\section{IIa Neutralization with Plasma; Computation Formalism}

To evaluated velocity space slowing down and diffusion formulas, which were derived from the test particle model ${ }^{10-14}$, are used in the next sub-section. As it was shown ${ }^{1}$ previously, physical parameters of this problem justify the use of formulas based on the test particle model. Hence, in this case the use of plasma physics formulas is fully justified to compute ion velocity space diffusion for the following reasons.

Given ion and electron beam parameters, the Debye length $\lambda_{D}=7.43 \times 10^{-4} \mathrm{~cm}$, hence there are about 1346 Debye lengths in a beam diameter. And, there are about 182 electrons in a Debye sphere. Electron gyro-radius is $3.13 \times 10^{-5} \mathrm{~cm}$. Hence, there are almost 32,000 electron gyro-radii in a beam diameter. Electron gyro-frequency is $6.72 \times 10^{10} \mathrm{~Hz}$ in this magnetic field of 2.4 Tesla. During an interaction time (computed above) of $\tau=$ $6 \times 10^{-8} \mathrm{sec}$, an electron completes $\mathbf{4 0 3 2}$ gyrations. No past, existing, or future (planned) electron beam cooler has parameters where beam diameter to gyro-radius and Debye length ratios, as well as the number of electron gyrations are such large numbers. Therefore, classical plasma physics formalism is fully justified in this analysis. In this magnetic field ion gyro-frequency is about $6 \mathrm{MHz}$, i.e. ion gyration period is over a factor of 5 larger than the longest interaction (cooling) time. So ions are not magnetized.

Rostoker and Rosenbluth ${ }^{10}$ developed the test particle model. Norman Rostoker continued to refine the test particle model in subsequent papers ${ }^{11,12}$ to a point where exact equations were written ${ }^{13}$ by Trubnikov for a Maxwellian field particle distribution.

Pertinent (to this case) relaxation rates $v^{\mathrm{p} / \mathrm{p}^{\prime}}$ in $\sec ^{-1}$ (p test particle in a background of field particles p') for slowing down, diffusion in velocity space perpendicular and parallel to the direction of the test particle motion are given in the following equations

$$
\frac{d}{d t} \overrightarrow{v_{p}}=-v_{s}^{p / p^{\prime}} \overrightarrow{v_{p}}
$$




$$
\begin{aligned}
& \frac{d}{d t}\left(\overrightarrow{v_{p}}-\overrightarrow{v_{p^{\prime}}}\right)_{\perp}^{2}=v_{\perp}^{p / p^{\prime}} \overrightarrow{v_{p^{\prime}}^{2}} \\
& \frac{d}{d t}\left(\overrightarrow{v_{p}}-\overline{v_{p}}\right)_{\|}^{2}=v_{\|}^{p / p^{\prime}} \overrightarrow{v_{p}^{2}}
\end{aligned}
$$

Velocities are denoted by $\mathrm{v}$ while rates are indicated by $v$. Subscripts $(s, \perp, \& \|)$ denote slowing down, transverse diffusion in velocity space and parallel diffusion in velocity space respectively. Averages are performed over an ensemble of test particle distributions for a Maxwellian field particle distribution. Exact formulas exist for relaxation rates ${ }^{13,14}$, which can be written as,

$$
\begin{aligned}
& v_{s}^{p / p^{\prime}}=\left(1+\frac{M}{m}\right) \psi(x) v_{0}^{p / p^{\prime}} \\
& v_{\perp}^{p / p^{\prime}}=2\left[\left(1-\frac{1}{2} x\right) \psi(x)+\psi^{\prime}(x)\right] v_{0}^{p / p^{\prime}} \\
& v_{\|}^{p / p^{\prime}}=[\psi(x) / x] v_{0}^{p / p^{\prime}}
\end{aligned}
$$

where $v_{0}^{p / p^{\prime}}=4 \pi \mathrm{Z}^{2} e^{4} \lambda n / M_{p}{ }^{2} v_{p}^{3} ; \mathrm{x}$ is essentially the ratio of the test particle energy to the field particle temperature. $\mathrm{Z}$ is ion charge state, e elementary charge and $\lambda$ is the Coulomb logarithm.

$\psi(x)=\frac{2}{\sqrt{\pi}} \int_{0}^{x} t^{1 / 2} e^{-t} d t$ and $\psi^{\prime}(x)=\frac{d \psi}{d x}$, (here e is not the elementary charge)

In cases where $\mathrm{x}>>1$ or $\mathrm{x}<<1$, (i.e. for very fast or very slow test particles) simpler limiting forms of the relaxation rates exist ${ }^{14}$. These equations are utilized in the next subsections for ion cooling and ion heating computations. For simplicity, computations are performed in the beam rest frame, since $\gamma=1.0022$, corrections to time dilations are minuscule.

Note that for relatively slow relaxation, equations 1-3 can be written as (for example equation 1)

$$
\Delta V_{p} / \Delta t \approx-v^{p / p^{\prime}} V_{p}, \text { hence } \Delta V_{p} \approx-v^{p / p^{\prime}} V_{p} \Delta t
$$

Depending on which formula is used for the Coulomb logarithm $\lambda$, on whether it is Parkhomchuk's ${ }^{15}$

$$
\lambda=\ln \left(\frac{\left[\frac{v_{t h i}}{\omega_{p e}}\right]}{\rho_{e}}\right) \approx 3.4
$$

or the expression derived by Montgomery et $\mathrm{al}^{16}$

$$
\lambda=\ln \left(\frac{\rho_{e}}{b}\right) \approx 3.2
$$


both yield similar numbers. Here $\omega_{\mathrm{pe}}$ and $\rho_{\mathrm{e}}$ are electron plasma frequency and gyroradius respectively; $b$ is the smallest impact parameter. In subsequent calculations $\lambda=3.3$ is used.

Finally, it is important to point out that the test particle model had experimental verification $^{17-19}$. Additionally, as part of the BNL neutral beam program (over 25 years ago) plasma devices ${ }^{20,21}$, with densities far exceeding those needed to neutralize the cooling electron beam, were successfully operated. And, successful neutralization experiments were performed at the BNL Tandem van de Graaff accelerator.

\section{IIb Velocity Slowing Down and Diffusion in Plasma}

First the effect of the background plasma on the gold ion beam is examined. To avoid the problem of charge exchange, neutralizing plasma should consist of protons and electrons (like hydrogen plasma generated in a hollow cathode arc). Start with listing of pertinent relaxation rates ${ }^{14}$ of gold ions on plasma ions (field particles are designated by prime) and plasma electrons.

$$
\begin{aligned}
& v_{s}^{i / i^{\prime}}=9 \times 10^{-8} \mu^{1 / 2} n Z^{2} \lambda \varepsilon^{-3 / 2}\left(1+\frac{1}{\mu}\right) \\
& v_{\perp}^{i / i^{\prime}}=1.8 \times 10^{-7} \mu^{-1 / 2} n Z^{2} \lambda \varepsilon^{-3 / 2} \\
& v_{\|}^{i / i^{\prime}}=9 \times 10^{-8} \mu^{1 / 2} n Z^{2} \lambda T_{i^{\prime}} \varepsilon^{-5 / 2}
\end{aligned}
$$

Units are cgs and $\mathrm{eV} ; \mu$ ion to proton mass ratio; $\varepsilon$ is beam particle energy; $v$ is in $\sec ^{-1}$. Ion beam slowing down and velocity space diffusion in a background of field electrons are given in the following equations.

$$
\begin{aligned}
v_{s}^{i / e^{\prime}} & =1.7 \times 10^{-4} \mu^{1 / 2} n Z^{2} \lambda \varepsilon^{-3 / 2} \\
v_{\perp}^{i / / e^{\prime}} & =1.8 \times 10^{-7} \mu^{-1 / 2} n Z^{2} \lambda \varepsilon^{-3 / 2} \\
v_{\|}^{i / / e^{\prime}} & =1.7 \times 10^{-4} \mu^{1 / 2} n Z^{2} \lambda T_{e} \varepsilon^{-5 / 2}
\end{aligned}
$$

Electron beam slowing down and velocity space diffusion on neutralizing plasma ions and electrons are given by the following equations.

$$
\begin{aligned}
& v_{s}^{e / i^{\prime}}=3.9 \times 10^{-6} n \lambda \varepsilon^{-3 / 2} \\
& v_{\perp}^{e / i^{\prime}}=7.7 \times 10^{-6} n \lambda \varepsilon^{-3 / 2} \\
& v_{\|}^{e / i^{\prime}}=2.1 \times 10^{-9} \mu^{-1} n \lambda T_{i^{\prime}} \varepsilon^{-5 / 2} \\
& v_{s}^{e / e^{\prime}}=7.7 \times 10^{-6} n \lambda \varepsilon^{-3 / 2} \\
& v_{\perp}^{e / e^{\prime}}=7.7 \times 10^{-6} n \lambda \varepsilon^{-3 / 2} \\
& v_{\|}^{e / e^{\prime}}=3.9 \times 10^{-6} n \lambda T_{e} \varepsilon^{-5 / 2}
\end{aligned}
$$


By inspection, it is clear from equations 5-10 that dominant effect on beam ions is slowing down on plasma electrons (equation 8). For our parameters $v_{s}^{i / e^{\prime}}=1.1 \times 10^{-1} \mathrm{sec}^{-1}$. Hence, from equation 4 (since cooling time $\tau=6 \times 10^{-8} \mathrm{sec}$ )

$\frac{\Delta v_{i s}}{v_{i}} \approx v_{s}^{i / e^{\prime}} \Delta t=6.6 \times 10^{-9}$

i.e. negligible compared to original ion momentum spread $\Delta p_{\|} / p=10^{-3}$, and $\Delta p_{\perp} / p=5 \times 10^{-3}$ or even after cooling momentum spread, which a factor of 12 smaller.

Repeating this process for electrons, it becomes obvious by inspection, of equations 1116 , that the dominant effect on beam electrons is perpendicular scattering by background plasma ion as well as slowing down and perpendicular scattering by background plasma electrons (equations 12, 14, and 15 respectively; also slow down by neutralizing plasma ions has an effect lower by a factor of 2). Since these effects can be some what additive, under the worse case scenario equation 4 yields for our parameters,

$\frac{\Delta v_{\text {etotal }}}{v_{e}} \approx 3.5 \bullet v_{s}^{e / e^{\prime}} \Delta t=1.7 \times 10^{-5}$

Comparing equation 18 to the compressed parallel thermal spread of beam electrons ${ }^{1}$ is about $5 \times 10^{-4} \mathrm{eV}$, hence the ratio of electron beam parallel spread to electron beam velocity is $\frac{\Delta v_{e \|}}{v_{e}} \approx \sqrt{\frac{T_{e \|}}{E_{e}}}=7 \times 10^{-4}$, which is an order of magnitude lower than equation 18 . In the perpendicular direction, there is no kinematic compression $\mathrm{T}_{\mathrm{e}}=0.1 \mathrm{eV}$, therefore any perpendicular diffusion in velocity space is orders of magnitude smaller (any effects contributed by equation 12 , could have been neglected).

\section{IIc Gas Neutralization}

Estimating the effect of low density neutral gas is more difficult, since the NIST ${ }^{22}$ CSDA range and stopping power can be found for energies no lower than $10 \mathrm{KeV}$. One way to estimate the energy loss is to calculate the values for $10 \mathrm{KeV}$ and extrapolate to $1 \mathrm{KeV}$. For hydrogen gas at STP $10 \mathrm{KeV}$ electrons have a range of about $1.3 \mathrm{~cm}$. Since 1 atmosphere has a density (at room temperature) of $2.736 \times 10^{19} \mathrm{~cm}^{-3}$, and since range is proportional to density, at a density of $1 \times 10^{11} \mathrm{~cm}^{-3}$ the CSDA range is $3.5 \times 10^{8} \mathrm{~cm}$, which can be extrapolated to $1 \mathrm{KeV}$ be $3.5 \times 10^{7} \mathrm{~cm}$. Total energy loss (of $1 \mathrm{KeV}$ electrons at a density of $1 \times 10^{11} \mathrm{~cm}^{-3}$ ) is $1.5 \times 10^{-4} \mathrm{eV} / \mathrm{cm}$, or $1.8 \times 10^{-2} \mathrm{eV}$ in $120 \mathrm{~cm}$. However, the dominant effect is lateral scattering of electrons ${ }^{23}$, which is a factor of $3-7$ larger. This energy broadening is still acceptable. For the gold ions it is not an issue.

Repeating the calculations for argon, the range of $1 \mathrm{KeV}$ electrons is $6.6 \times 10^{6} \mathrm{~cm}$. Total energy loss comes to be close to $0.1 \mathrm{eV}$, hence lateral scattering could lead to perpendicular energy broadening of about $0.5 \mathrm{eV}$. Hence neutralization with argon gas is marginal at best especially when charge exchange ${ }^{1}$ with beam ions is considered. 


\section{Pertinent Physics Issues}

A number of pertinent physics issues were evaluated in previous notes ${ }^{1}$. Those analyses are still valid. Due to the background plasma, the electron beam and to a much lesser extend the ion beam are now potential sources of free energy. Therefore, electron beam stability due to beam - plasma and anisotropic electron beam temperature must be looked at, even though the conditions are low densities (of $1 \times 10^{11} \mathrm{~cm}^{-3}$ ) and an axial magnetic field of 2.4 Tesla. Like all beam instabilities, the first has a density threshold and it is suppressed by the very large magnetic field. It's possible that in order to prevent beamplasma instability, the neutralizing plasma density must be raised. In that case, electron beam velocity slowing down must be re-evaluated. The second instability is a hydromagnetic instability, i.e. a fluid instability. The densities in question are well below conditions required for fluid treatment of plasmas. Furthermore, the short interaction time would prevent any substantial growth.

\section{Higher Energy Injection}

Magnetically confined electron beams have longitudinal transport limits. Similar to the Child Langmuir law in one dimension, there is a three-dimensional equivalent problem ${ }^{7}$ in propagating high-current electron beams through a cylindrical conducting vacuum tube with a strong axial solenoidal magnetic field. On axis potential $\varphi$ for uniform spacecharge density is given ${ }^{7}$ by

$\phi=\frac{30 I_{0}}{\beta}\left[1+2 \ln \left(\frac{r_{w}}{r_{0}}\right)\right]$

where $\mathrm{r}_{\mathrm{w}}$ and $\mathrm{r}_{0}$ are chamber wall and electron beam radii respectively. For $\mathrm{r}_{\mathrm{w}} / \mathrm{r}_{0}=1.1(\beta=$ 0.0667 ), equation 19 yields about $13.3 \mathrm{KV}$ for $\varphi$, which means that the electron beam entering the interaction region must have at least $14.3 \mathrm{KV}$. For $\mathrm{r}_{\mathrm{w}} / \mathrm{r}_{0}=1.2, \varphi$ is about 15.3 $\mathrm{KV}$, hence at least $16.3 \mathrm{KV}$ is needed.

Based on equation 19, reference 7 gives examples of why induction LINAC injectors must operate at higher energies to compensate for beam slowed by the potential. The claim of at "least $16.3 \mathrm{KV}$ " is rather crude, since it implies relatively small change in $\beta$, which is not the case.

A more exact estimate of the axial potential can be computed from ${ }^{7}$

$$
\frac{1}{r} \frac{d}{d r}\left[r \frac{d \phi}{d r}\right]=\frac{j_{0}\left(1+e \phi / m c^{2}\right)}{c \varepsilon_{0}\left[2 e \phi / m c^{2}+\left(e \phi / m c^{2}\right)^{2}\right]^{1 / 2}}
$$

Obviously, equation 20 is very convoluted. A long iterative numerical process is required $^{7}$ to solve equation 20 . Based on a solution, the needed electron beam injection is the potential computed from equation 20 plus $1 \mathrm{kV}$, i.e. electron beam injection energy is $\varphi+1 \mathrm{KV}$.

In the limit of an electron beam radius, which is much smaller than the wall, there is a simple expression for computing the required injection energy $\gamma_{0}$, which is given ${ }^{7}$ by 
$\left[\frac{e I_{0}}{4 \pi \varepsilon_{0} m c^{3}}\right]\left[1+2 \ln \left(\frac{r_{w}}{r_{0}}\right)\right]=\frac{\left(\gamma_{0}-\gamma\right)(\gamma-1)^{1 / 2}}{\gamma}$

Assuming that $r_{w} / r_{0}=10$, for final electron beam energy of $1 \mathrm{KeV}(\gamma=1.0022), \gamma_{0}$ of the needed injection energy is 1.1775 , i.e. $\beta=0.528$ or about $71.5 \mathrm{KV}$ is needed. The above results are for $\mathrm{I}_{0}=25 \mathrm{~A}\left(\mathrm{j}_{0}=31.8 \mathrm{~A} / \mathrm{cm}^{2}\right)$. With greatly reduced conduction wall effect; the space-charge problem is increased.

This voltage level is easily achievable. And, interesting possibilities open up. Based on the Child Langmuir law $j=2.33 \times 10^{-6} \frac{V^{3 / 2}}{d^{2}}$, at $71.5 \mathrm{kV}$ e.g., for a gap of $0.5 \mathrm{~cm}$, about $178 \mathrm{~A} / \mathrm{cm}^{2}$ can be extracted for that voltage $\left(31.8 \mathrm{~A} / \mathrm{cm}^{2}\right.$ is needed). It implies that Conventional thermionic cathodes could be used.

This type of slowing down may result in instabilities. A variation on this idea is to injected high energy electrons into a negatively biased cooling section, i.e. into a biased drift tube. Electrons can be extracted at the Child Langmuir limit. The electrons slow down as they enter the drift tube, while ions are accelerated. In principle, electron injection energy and a drift tube bias voltage, which results in matching electron and ion drift velocities, can be found; work in progress!

\section{Conclusion}

Plasma neutralizer parameters ${ }^{20,21}$ a meter long $\mathrm{n}>10^{13} \mathrm{P}=10^{-6}$; induction LINAC injector ${ }^{7}$. Other than Parkhomchuk's empirical formula, which is used to calculate cooling rates, most computations in this note are based on plasma physics formalism. Given ion and electron beam parameters, the Debye length $\lambda_{D}=7.43 \times 10^{-4} \mathrm{~cm}$, hence there are about 1346 Debye lengths in a beam diameter. And, there are about 182 electrons in a Debye sphere. Electron gyro-radius is $3.13 \times 10^{-5} \mathrm{~cm}$. Hence, there are almost 32,000 electron gyro-radii in a beam diameter. Electron gyro-frequency is $6.72 \times 10^{10} \mathrm{~Hz}$ in this magnetic field of 2.4 Tesla. During an interaction time (computed above) of $\tau=$ $3.35 \times 10^{-8} \mathrm{sec}$, an electron completes 2251 gyrations. In this magnetic field ion gyrofrequency is about $6 \mathrm{MHz}$, i.e. ion gyration period is over a factor of 5 larger than the longest interaction (cooling) time. So ions are not magnetized. No past, existing, or future (planned) electron beam cooler has parameters where beam diameter to gyro-radius and Debye length ratios, as well as the number of electron gyrations are such large numbers. Furthermore, transport and velocity space relaxation theories based on the test particle model were proven to be correct experimentally ${ }^{23}$ in a series of experiments performed on two different devices. Therefore, classical plasma physics formalism, especially when based on the test particle model, is fully justified in this analysis.

Answer to the question posed in the title, on whether single pass cooling is possible, is affirmative. While velocity relaxation and cooling computations, based on the test particle model, have had experimental verification, electron beam cooling theories did not agree with cooling experiments. Hence, the need for Parkhomchuk's empirical 
formula ${ }^{10}$, which has shown to be in good agreement with ion cooling (slowing-down) computations (equations 11 and 18) that are based on the test particle model. Some discrepancies with theories used in conjunction with electron beam cooling may be due to the very different parameters of this case as compared to parameters in electron beam cooling. In equation 15, e.g. the electron beam temperature (instead of a delta function) must be included due to overlapping of ion and electron distributions in velocity space.

Further evaluation requires an iteration process, of simulations and of electron gun as well as electron beam cooler design, to further explore concepts presented in this note. Electron guns with carbon fiber cathodes should be able to achieve the needed electron beam parameters. Carbon nano-tubes might be superior, due to their extreme durability, which also eliminates any gas problems.

Based on cooling computations performed in sub-sections IIIa and IIIb, momentum spread of gold ion beams exiting the EBIS LINAC can be reduced by a factor of about 14 in a cooling distance of $107 \mathrm{~cm}$ (based on plasma physics formalism) or $120 \mathrm{~cm}$ (Parkhomchuk's empirical formula).

During a talk given by the author on $1 / 23 / 2009$, Alexei Fedotov ${ }^{24}$ pointed out that he had just repeated the cooling calculation (using Parkhomchuk's empirical formula) and found agreement with reference 1 calculations, when only ion velocity spread is considered. Inclusion of space charge effects on electrons increases the cooling length by a factor of 2 - 3; still not a show stopper. However, when space charge effects on the ions are considered, the cooling length increases to about $2-3 \mathrm{~km}$; definitely a show stopper! Subsequently, a possible solution was found: co-injection of $2 \mathrm{MeV}$ protons from a proton diode similar to those generated at $\mathrm{NRL}^{25}$, but with higher energy than their 1.2 $\mathrm{MeV}$, and much lower current than their $1 \mathrm{MA}$ (should be a relatively straight forward task). Co-injection of equal $2 \mathrm{MeV}$ proton current will solve the space-charge problem associated with the electron beam.

Interesting physics regardless of the particular application! Motivation for this work is indeed cooling the EBIS LINAC ion beams before injection into the Booster. However, as initial calculations were performed it became apparent that there is a consensus in the electron beam cooling community that single pass cooling is impossible. If successful it will $1^{\text {st }}$ single pass cooling ever with implications far beyond this particular case.

\section{References}

1. Ady Hershcovitch, "Emittance Reduction between EBIS LINAC and Booster by Electron Beam Cooling; Is Single Pass Cooling Possible?" C-A/AP note \#303 April 2008 (unpublished); Ady Hershcovitch, "Part II/Addendum Electron Beam Cooling between EBIS LINAC and Booster; Is Single Pass Cooling Possible?" CA/AP note \#317 July 2008 (unpublished).

2. Deepak Raparia, private communication (2008).

3. Ady Hershcovitch, APL 68, 464 (1996).

4. A. I. Hershcovitch, V. J. Kovarik, and K. Prelec, J. Appl. Phys. 67, 671 (1990). 
5. A. Hershcovitch, J. Appl. Phys. 74, 728 (1993).

6. A. Hershcovitch, "Proceedings of the Fifth International EBIS Symposium", edited by E. D. Donets and I. P. Yudin (JINR, Dubna, EBIS-5, Russia, 1991).

7. S. Humphries Jr., "Charged Particle Beams", John Wiley and Sons Inc., New York (1990).

8. G. R. Brewer, "High Intensity Electron Guns \& Focusing of High Density Electron Beams", in "Focusing of Charged Particle", Volume 2, A. Septier, Editor, Academic Press, New York (1967).

9. Alexander Pikin, private communication (2008).

10. N. Rostoker and M.N. Rosenbluth, Physics of Fluids 3, 1 (1960);

11. N. Rostoker, Nuclear Fusion 1, 101 (1961).

12. N. Rostoker, Physics of Fluids 7, 491 (1964).

13. B.A. Trubnikov, Reviews of Plasma Physics, Vol. 1, (Consultants Bureau, New York, 1965), pp. 105-204.

14. NRL Plasma Formulary, J. D. Huba, NRL/PU/6790-06-491, revised 2006.

15. V.V. Parkhomchuk, NIM A 441, 9 (2000).

16. D. Montgomery, G. Joyce, and L. Turner, Physics of Fluids 17, 2201 (1974).

17. J. Bowles, R. McWilliams, and N. Rynn, Phys. Rev. Letters 68, 1144 (1992).

18. J. Bowles, R. McWilliams, and N. Rynn, Physics of Plasmas 1, 3418 (1994).

19. J.J. Curry, F. Skiff, M. Sarfaty, and T.N. Good, Phys. Rev. Letters 74, 1767 (1995).

20. A. Hershcovitch and V.J. Kovarik, Rev. Sci. Instrum. 54, 328 (1983).

21. A.I. Hershcovitch, B.M. Johnson, V.J. Kovarik, M. Meron, K.W. Jones, K. Prelec, and L.R. Grisham, Rev. Sci. Instrum. 55, 1744 (1984).

22. The NIST website http://physics.nist.gov/PhysRefData/Star/Text/

23. A. Hershcovitch, J. Appl. Phys. 78, 5283 (1995); A. Hershcovitch, Phys. Plasmas, 5, 2130 (1998). Expressions derived from H.A. Bethe's work and are based on formulas, which can be found in text books, e.g., J.D. Jackson, "Classical Electrodynamics", Second Edition, Wiley, New York, 1975.

24. Alexei Fedotov private communication 2009.

25. Goldstein et al, PRL $\underline{40}, 1504$ (1978); Young et al, PRL $\underline{70}, 2573$ (1993).

\section{Acknowledgement}

Very helpful discussions with Deepak Raparia, Ilan Ben-Zvi, Brant Johnson, Norman Rostoker and Roger McWilliams are gratefully acknowledged. Many thanks to Alexei Fedotov for numerous stimulating and extremely helpful discussions!

Notice: This manuscript has been authored by Brookhaven Science Associates, LLC under Contract No. DE-AC02-98CH1-886 with the US Department of Energy. The Untied States Government retains, and the publisher, by accepting the article for publication, acknowledges, a world-wide license to publish or reproduce the published form of this manuscript, or others to do so, for the United States Government purposes. 